\title{
Detection of myocardial edema with diffusion weighted imaging in patients with acute myocarditis
}

\author{
Anna Kociemba1*, Justyna Rajewska-Tabor ${ }^{1}$, Magdalena Lanocha' ${ }^{1}$, Magdalena Janus ${ }^{1}$, Andrzej Siniawski ${ }^{1}$, \\ Katarzyna Karmelita-Katulska², Magorzata Pyda' \\ From 18th Annual SCMR Scientific Sessions \\ Nice, France. 4-7 February 2015
}

\section{Background}

Diffusion weighted imaging has been shown to be promising method for myocardial infarction, myocarditis, and myocardial fibrosis imaging. The aim of this study was to evaluate oedema with low $b$-value $\left(b=50 \mathrm{sec} / \mathrm{mm}^{2}\right)$ diffusion-weighted imaging (DWI) and compare it with routinely used "Lake Louise Criteria" in patients with acute myocarditis.

\section{Methods}

We have analyzed 26 consecutive patients: 22 male, average age: 27 years (range 13-43) with clinical diagnosis of acute myocarditis. The CMR examinations were performed on a 1,5 $\mathrm{T}$ scanner using an eight-channel phasedarray coil combined with 4-6 elements of spinal coil. All patients underwent assessment of myocardial oedema: $\mathrm{T}_{2^{-}}$ weighted triple inversion recovery (STIR), $\mathrm{T}_{1}$-weighted turbo spin echo pre and post contrast, function (cine Steady State Free Precession) and scar (Late Gadolinium Enhancement). Additionally DWI EPI sequence with $b=$ $50 \mathrm{sec} / \mathrm{mm}^{2}$ was acquired before contrast administration. The sequence parameters were as follows: slice thickness $10 \mathrm{~mm}$, repetition time (depending on patient breath cycle) 3-4 s, echo time $78 \mathrm{~ms}$, bandwidth $1,736 \mathrm{~Hz} / \mathrm{Px}$. The DWI sequence was ECG-gated and synchronized to the respiratory cycle using PACE technique. For all patients $\mathrm{T} 1$ and $\mathrm{T} 2$ ratio were calculated and presence of LGE areas were reported. For STIR and DWI contrast between healthy myocardium and edema was calculated as a difference between edematous and normal myocardial muscle divided by standard deviation of image noise.

I'st Department of Cardiology, Magnetic Resonance Unit, University of Medical Sciences in Poznan, Poznań, Poland

Full list of author information is available at the end of the article

\section{Results}

We managed to acquired good quality DWI images in all 26 patients, average acquisition time was 120 s per slice, distortion artifacts occurred in 5 patients but did not impaired diagnostic value of analyzed images. Increased signal intensity in DWI images occurred in all patients in the area of LGE enhancement and were consistent with areas of increased signal in STIR. All patients met at least two out of three criteria for inflammatory activity and injury. $T_{2}$ ratio was increased $(\geq 2)$ in 24 patients, $T_{1}$ ratio $(\geq 4)$ in 23 cases, all patients had focal non ischemic enhancement in LGE. CNR was higher in DWI than in STIR: 23,8 vs. 17,6 respectively.

\section{Conclusions}

DW EPI is a promising sequence for myocardial oedema detection in patients with acute myocarditis and may be useful in everyday clinical practice.

\section{Funding}

Not applicable.

\section{Authors' details}

'I'st Department of Cardiology, Magnetic Resonance Unit, University of Medical Sciences in Poznan, Poznań, Poland. ${ }^{2}$ Department of General Radiology, University of Medical Sciences in Poznan, Poznan, Poland.

Published: 3 February 2015

doi:10.1186/1532-429X-17-S1-P331

Cite this article as: Kociemba et al:: Detection of myocardial edema with diffusion weighted imaging in patients with acute myocarditis. Journal of Cardiovascular Magnetic Resonance 2015 17(Suppl 1):P331. 